\title{
Minimizing the Energy Consumption Using a Secure Recursive Localization Approach for Distributed Networks in Underwater Acoustic Wireless Sensor Networks
}

\author{
Vaishnavi Shukla $^{1^{*}}$, Awadhesh Kumar ${ }^{2}$, Vijay Bhan ${ }^{3}$ \\ ${ }^{1}$ Department of CSE, Kamla Nehru Institute of Technology Sultanpur, AKTU, Uttar Pradesh India \\ ${ }^{2}$ Department of CSE, Kamla Nehru Institute of Technology Sultanpur, AKTU, Uttar Pradesh India \\ ${ }^{3}$ Department of Computer Science and Engineering, NIT Rourkela, Orissa, India \\ *Corresponding Author: vaishnavishukla@mpgi.edu.in, 8565808849
}

Available online at: www.isroset.org

Received: 25/May/2018, Revised: 11/Jun/2018, Accepted: 23/Jun/2018, Online: 30/Jun/2018

\begin{abstract}
In wireless sensor networks, Underwater Acoustic Sensor Network (UASN) is a network of wireless sensor nodes dispensed upon an absolute area within an underwater environment, communicating along each mean via acoustic signal. It executes action as much as helping technology because a variety over underwater applications. In spite about the records centric behaviour on the UASN, on occasion the accumulated facts except its area of occurrence emerge as meaningless. Thus, localization for UASNs has turn out to be an absolutely excellent theme of research. This paper proposes an event-driven distributed localization scheme for short distance 2D UASN. The Proposed design employs a recursive localization manner of which efficiently localized nodes can work as reference node to aid within localization over the other ordinary sensor nodes. The proposed scheme employs a recursive localization procedure in which we can improve better power consumption, packet delivery ratio and delay. The simulation is done using Network Simulator-2.35.
\end{abstract}

Keywords-Anchor nodes, Acoustic Signal, Localization, UASN, Energy model, AODV, Trust Value.

\section{INTRODUCTION}

Wireless sensor network (WSN) are widely used for various applications and sensors are deployed such as in healthcare monitoring, military surveillance and many new exiting fields. This all has been possible due to recent technological advancement which has been led to development of cheap and low power sensors. These sensors can sense various environmental activities such as temperature, pressure, sound etc. The sensor node has very low computability as they operate on low power budget.

The main work of wireless sensor network is to accumulate, monitor and forward data to destination. Sensor nodes are deployed in area where we want to track the activities such as in forest to monitor forest fire or to track the wild life, military application for international border or for tracking enemy activities or in unmanned machines, tracking of bridges for monitoring of their structural health. In some cases where weather condition is abnormal it is difficult to provide a constant power source to sensor nodes in hostile terrains. It is difficult to extract the sensed data in such environment because sensor nodes used their energy very judicially there. Operation can also be less performed in sensed data. The sensed data is then sent to base station for further processing. Determining the physical location of sensor node is called problem of localization. Such type of problem also held in underwater environment and such a network is called Underwater Acoustic Sensor Network (UASN). Thus, localization of nodes of underwater networks is very interesting topic of research. Ocean covers about $70 \%$ area of the whole earth surface. Ocean energy which is great source of renewable energy through which electricity can be generated with the assistance of tidal waves and it is rich in large number of valuable resources which took place consideration of human to surveyed and make research in ecological environment. There is huge number of sensor nodes which are deployed in underwater just to accomplish a specific work. These sensor nodes form a network inside and coordinating amongst these nodes with each other can used to sense environmental phenomena in distributed ways.

Underwater Networks forms a various type of applications that include ocean sampling, monitoring environmental activities, ocean exploitation for scientific as well as commercial purposes, navigation assistance, disaster management etc. Localization is the phenomena through which we are able to find out the physical coordinates, i.e. position of sensor node in underwater environment. Acoustic signal is the best way of communication among the objects within the underwater surroundings due to the fact radio signal attenuates very high and optical signal scatters. Hence, UWSN redefined newly as Underwater acoustic sensor network i.e. UASN. 


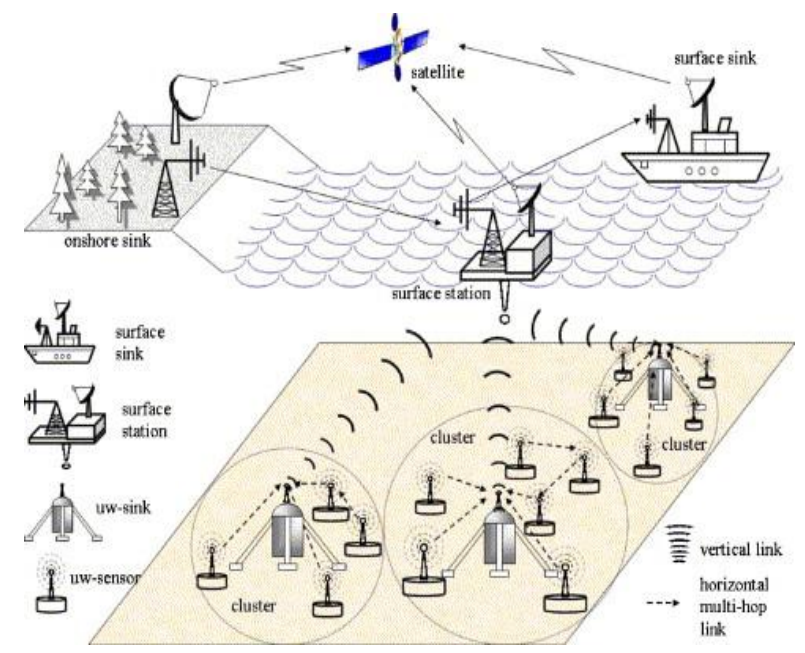

Fig. 1 Architecture of underwater sensor networks

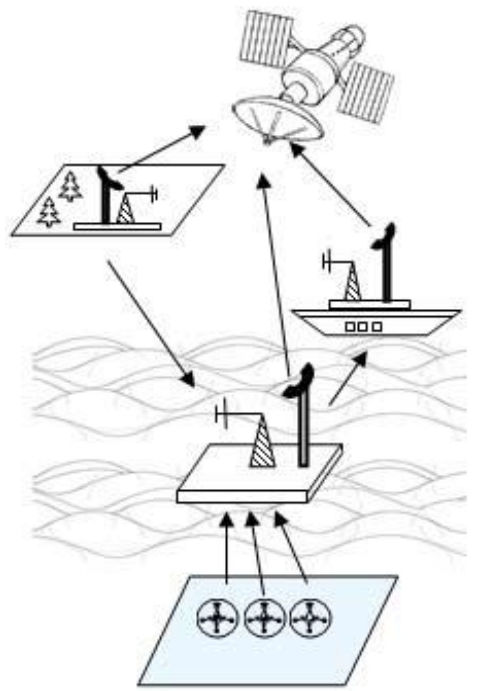

Cluster

Surface Sink

Surface Station

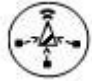

Fig.2 2-D Architecture of UWSN

The Underwater acoustic communication is a process in which we can send and receive message under the water. There are so many techniques to employ such type of communication yet very used to technique is communication done via. hydrophones. There are some facts because of that Underwater communication is difficult are multi-path propagation, time variants of the channel, little available bandwidth then vivid signal attenuation, especially on long ranges. In terrestrial communications, the data charges are excessive as compared after underwater communication due to the fact underwater communication usage acoustic waves instead of electromagnetic waves. Even today in underwater networks many problems have been seen such as in localization of nodes. Challenges of such networks are energy consumption, battery consumption, cost, power, deployment of nodes, routing, bandwidth, storage capacity.
The underwater acoustic WSN are shown in figure 1 and 2D Architecture of underwater sensor network is shown in fig. 2 . The following work is organized as described below. The introduction of underwater sensor network is defined in Section I related works and terminologies related to it are presented in Section II and Section III defines methodology respectively. The result and analysis of UWSN is discussed and analyzed in the Section IV and finally, in Section V conclusion is described.

\section{RELATED WORK}

\section{TERMINOLOGIES USED IN LOCALIZATION OF NODES}

Anchor nodes: Some nodes are distributed in a network which is already knows their location such types of nodes are called Anchor nodes. These nodes are used for estimating the position of unknown nodes or ordinary nodes. In simulation of wireless networks, the location of these types of nodes provides manually and they acquire more power than the ordinary nodes.

Reference nodes: After localization scheme applied on unknown nodes to find their location some nodes are get localized and these nodes are used to estimate distance of other nodes which are called reference nodes.

Ordinary nodes or Unknown nodes: These are the nodes which have to be localized. The main motive of our project is to localize these types of nodes, to reduce power consumption, high PDR. First, we provide manually location to some nodes which are called beacon nodes after that by applying efficient localization algorithm we get some reference node after that these nodes are used for calculating distance of unknown nodes through which we find exact location of unknown node. Unknown nodes consumed less power or energy compare to beacon nodes.

The related works which are surveyed previously are discussed as:

In [1], paper defines a layout of an automatic system which is used to check or observe the sound and pressure generated by using anthropogenic activities. These things to do are such as underwater destruction and opile driving. These systems are organized such that it can record acoustic signal. The underwater sound recording machine (USR) permits for connections of joining hydrophones then other potential pressure sensors, filters excessive frequency noise abroad of the accumulated signals, has a gain so much execute stand independently engage because of every sensor, and permits because 2 hour of information collection. Two versions regarding the USR have been created: a durability submersible model deployable after a most deepness regarding $300 \mathrm{~m}$, or a watertight but no longer completely submersible model. 
[2], Researches are broadly used of deep applications, yet many studies have been conducted. However, nearly all modern research initiatives have not instituted network safety of consideration, in spite of the main truth is that a UASN is usually susceptible to malicious attacks appropriate according to the special traits of an underwater acoustic communication channel (e.g., low communication bandwidth, lengthy propagation delays, yet excessive bit error rates). In addition, the huge variations between UASNs and earthly wireless sensor networks entail the urgent then excessive fast development about proof communication mechanisms because of underwater sensor nodes. Due to the above observed caused, it composition goals according to current a extremely comprehensive land survey concerning the rising topics arising beyond impervious communications between UASNs, who naturally lead to a vast range on start lookup issues outlined afterward. The main work which is accomplished with the help of this article are defined as follows:

- The secure communication protocols between each tier for UASNs are diligently analyzed within the article. To the good about our knowledge, that is the advance work according to study invulnerable communication because of UASNs seam by layer. - The overall performance regarding the present impervious communication protocols is highlighted among phrases about their strengths then weaknesses. In addition, the malicious attacks then their countermeasures in each layer are identified.

[3], This paper present an acoustic transmitters as have been raised due to the fact makes usage of between underwater neutrino telescopes are presented. Firstly, an acoustic transceiver has been raised specifically stability portion over the acoustic positioning regulation concerning neutrino telescopes. Secondly, a shut acoustic transmitter array has been superior due to the fact of the calibration in relation to acoustic neutrino discovery systems.

Underwater Wireless Sensor Networks (UWSNs) are widely back according to discovery used aqueous environment. In UWSN, figuring out the location over sensor nodes is a crucial issue. Sensed record is significant only when sensing node is localized. There are many methods on hand because of localization within Wireless Sensor Network (WSN) but they are not relevant in UWSN. GPS signals cannot be used underwater for localization. Underwater communication is based totally on acoustic waves. This paper explores the different localization schemes accessible for UWSN and challenges according to meet the localization issue. Node mobility, high propagation delay, time synchronization yet excessive bit error rate are the most important challenges that want to be addressed [4].

Deployment of nodes in wireless Ad-hoc networks is very easy and compatible. But, ignoring this there is various security problems are came into existence and the most worth problem are interoperability and in communication for the management and configuration of various security issues. The packets drop ratio and energy consumption in the wireless network because sometimes it may be delayed by the attackers by dropping the sending packet. It may also cause delay in the WSN. Hence, some intruders detection techniques is used which may cause detection of intruder and minimizing the delay and packet drop ratio of nodes in wireless sensor networks.

In this paper author uses the trust value for the detection of intruders or malicious activity of attackers. They used inbuilt ids of the nodes and forward packet with the help of some another paths from that attacker is get confused and they cannot harms sending packets in networks [13].

Underwater sensor networks is successful according to perform operations in huge extent about applications up to expectation application operate one of a kind within USN partial applications likes distributed tactical surveillance, mine reconnaissance, sea sampling networks , seismic monitoring, environmental monitoring, tools monitoring, Disaster prevention, assisted Navigation yet undersea explorations this whole are the advantages regarding the underwater sensor networks. An efficient approach over communication has been adopted into the block zone via the usage of the combination regarding adaptive duty cycle or Network coding approach. Energy efficiency of the bottleneck zone will come extended due according to more volume on the statistics that would keep transmitted to the sink with the equal variety over transmissions. Hence the existence on the underwater sensor network is increased. This action archive to the enhancement on the underwater network life by means of considering a community coded adaptive duty cycle WSN. By applying the above strategies we finally acquire the enhance throughput of nodes. This proposed system investigates life time improvement approx $8 \%-14 \%$, yet minimizing power consumption. This paper is additionally referring to as useful because of imparting brief overview respecting each yet each and every protocol then accountable for entire underwater wireless sensor networks [14].

The Ad-hoc On-Demand Distance Vector (AODV) routing protocol is designed for the use in ad-hoc mobile networks. Ad-hoc networks are the one which made at instant of time or we can say spontaneous. AODV is a reactive protocol: the routes are tooled only when they are needed. It uses traditional routing tables, one access by destination, yet sequence numbers to determine whether routing information is up to date or to stop routing loops. An vital feature concerning AODV is the upkeep of time-based states of every node: a routing-entry not currently used is expired. In suit concerning a route is broken the neighbors can remain notified. Route search is based totally on query yet reply cycles, yet route statistics is stored among every intermediate nodes along the route between the form regarding path table entries. The consequent control packets are used: routing 
request message (RREQ) is broadcasted through a node requiring a path after another node, routing reply message (RREP) are unicasted and returned to the source of RREQ, then path error message (RERR) is dispatched according to information of other nodes about the impairment over the link. HELLO messages are back because of detecting or monitoring links to neighbors [15].

In this section, the author describes the previous research works in the form of title, problem statement, objectives, not repeat the information discussed in Introduction [3].

\section{METHODOLOGY}

Our Implementation algorithms had been carried out as: Hash-Based Message Authentication Code (HMAC) HMAC is a mechanism which used because for message authentication along the help of cryptographic hash functions, such a name as like MD5 or SHA-256, within aggregate together with a secret shared key. Our implementation makes usage regarding the available implementations for the underlying hash functions and needs only to connect the resulting byte streams effectively according to the protocol security for location algorithms. The proposed protocol is based about an shared secret key technology. An approaches is assumed to set up pair-wise secret keys. Source and Destination nodes each are not compromised. AODV routing protocol method up to expectation bi-directional links which means if a node $\mathrm{A}$ is able to acquire packet transmitted directly by means of some node $\mathrm{d}$ after node $\mathrm{d}$ is also capable of receiving packet directly transmitted via node $\mathrm{c}$. The notation used to describe cryptography operations is as follows. $\mathrm{C}$ and $\mathrm{f}$ are source and destination nodes respectively. KS denotes the secret key shared between nodes E and S. Each node holds the MAC (hash function-based message authentication) algorithm. The Hash functions strategies denote the computation of the message authentication security code of message $\mathrm{N}$ using secret key KS among nodes $\mathrm{c}$ then $\mathrm{f}$. The process of implemented algorithm will be defining as:

1. Starting process of the communication are between 2 nodes. Client send the request the server side and server send the acknowledge. Transmission will be done between Network Manager and 0 node 1 is a intermediate node between trasnmission process.

2. The communication in WSN network starting to sending and receiving the Data using authentication process generated secret key, issuing the tickets.

3. The packet drops during communication process between network manager and a WSN node. When client generate the request message to server then server generate the reply message within same window size. If that time client drops the packet that means, it's a suspicious node.
4. After that the authentication process is completed after then generates a request message to issuing the ticket or certificate authentication. Node requests a secret key to generate a request of a valid local $\mathrm{CA}$ or ticket. The node 0 Authentication process of a node and checking its corresponding identity node and if authentication process is completed then network manager generates a secret key of a node 0 .

5. After completion the Trusted or un trusted process node network manager issue a ticket, and authenticated node send the service request for a valid session key.

6. The process of Registration, Authentication, Validation, trusted node all process is completed then generates a valid key session.

The simulation parameters used in algorithms are defined with the help of given table (1).

\section{RESULTS AND DiscuSSION}

The proposed work is simulated with the help of NS-2.35. The simulation parameters will be given in table-1. The simulation table defines various parameters and regarding that values are defined.

Simulation can be done using 3 beacon nodes and $20,40,60,80,100$ unknown nodes. The simulation area is $2000 * 2000$ meter square. The AODV routing protocol can be used to route the hops using physical and wireless network interface. The packet size of network is 512 .

As we discuss in proposed algorithm we use energy model and secure hash algorithm in which we try to improve energy, delay, packet delivery ratio.

The network model is defined in which deployment of 20 nodes, communication of nodes and range will be defined are shown in figure 3 . The communication is done between each and every node deployed and defines our range with the help of circles after that they find there next hop which have highest energy. This animator also shows there packet drop.

Table-1 Simulation table

\begin{tabular}{|c|c|}
\hline PARAMETERS & VALUES \\
\hline Operating System & Linux (Ubuntu 12.04) \\
\hline NS-2 version & NS-2.35 for IEEE 802.11 \\
\hline No. of Nodes & $20,40,60,80,100$ \\
\hline Number of Unknown Node & 1 \\
\hline Number of beacon Node & 3 \\
\hline Radio propagation model & Propagation/TwoRayGround \\
\hline Network interface type & Phy/WirelessPhy \\
\hline Packet Size & 512 \\
\hline Traffic Type & UDP-CBR \\
\hline Execution Time & 200sec \\
\hline Antenna Type & Omni-Antenna \\
\hline
\end{tabular}




\begin{tabular}{|c|c|}
\hline Transmission Range & $2000 * 2000 \mathrm{~m}$ \\
\hline Transmitting Power & 0.281838 \\
\hline Power's watt & 0.281838 \\
\hline Receive Threshold & $7.69113 \mathrm{e}-08$ \\
\hline Carrier Sense & $5.3352 \mathrm{e}-6$ \\
Threshold & 0.5 \\
\hline Initial Energy & $\begin{array}{c}\text { Delay, Energy, Throughput, } \\
\text { PDR }\end{array}$ \\
\hline Performance parameters & $\begin{array}{c}\text { AODV,CA, Hash function, } \\
\text { Energy Consumption Model }\end{array}$ \\
\hline Routing Protocol (Proposed)
\end{tabular}

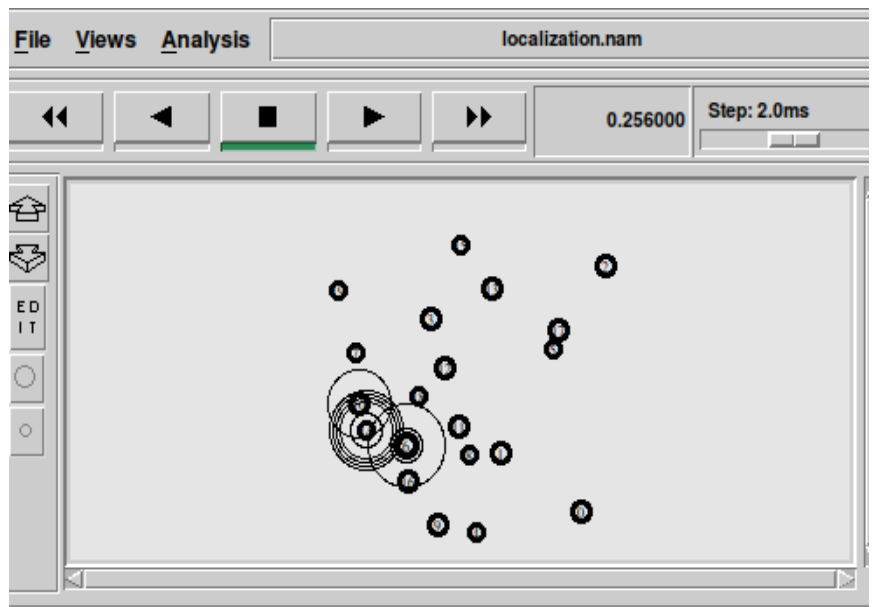

\begin{tabular}{|c|l|l|}
\hline No. Of nodes & Base technique & Proposed work \\
\hline 20 & 139 & 82 \\
\hline 40 & 305 & 4952 \\
\hline 60 & 4952 & 4952 \\
\hline 80 & 4952 & 4952 \\
\hline 100 & 4952 & 4952 \\
\hline
\end{tabular}

Table-3 Node vs. Received packet

\begin{tabular}{|l|l|l|}
\hline No. Of nodes & Base technique & Proposed work \\
\hline 20 & 0 & 43 \\
\hline 40 & 25 & 121 \\
\hline 60 & 1340 & 4812 \\
\hline 80 & 1227 & 3516 \\
\hline 100 & 1889 & 3469 \\
\hline
\end{tabular}

The average time that is taken by a data packet to reach to its destination. This includes all possible delays triggered by using buffering during route discovery latency, queuing at the interface queue. This metric is deliberated by taking the difference of the time at which the first packet is being transmitted by the source to the time at which first data packet arrived to destination.

It can be calculated with the help of following formula:

\section{Avg. $\mathrm{EED}=\mathrm{S} / \mathrm{N}$}

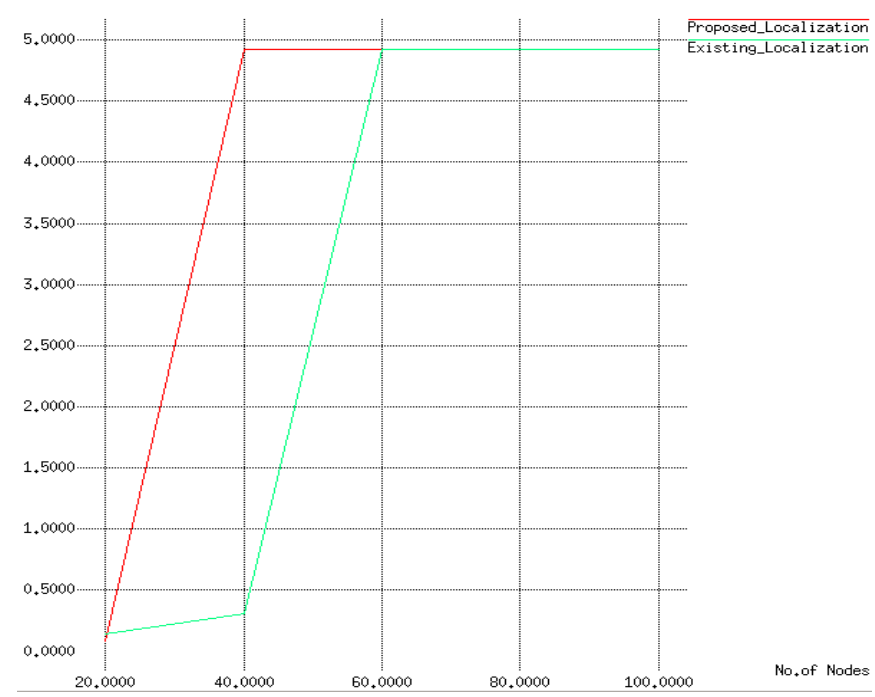

XGraph-2 Received packet vs. Node

Where, $\mathrm{S}$ is the summation of the time which spends in delivering the packets to each destination, and $\mathrm{N}$ is defined as the number of packets that is received by each destination nodes. After calculation of received packet we calculate the delay in packet received that is defined by taking difference 
of end time of node and start time of node are shown in table 4.

Table-4 Node vs. Delay

\begin{tabular}{|l|l|l|}
\hline No. Of nodes & Base technique & Proposed work \\
\hline 20 & 0 & 344.36 \\
\hline 40 & 1.89 & 192.15 \\
\hline 60 & 190.14 & 145.75 \\
\hline 80 & 595.62 & 347.36 \\
\hline 100 & 625.33 & 440.95 \\
\hline
\end{tabular}

Now, we calculate energy of nodes by the formula-

\section{Energy $=$ Sum of $x$ and $y$ coordinates/Total no. Of nodes}

But the difference between implemented and previous one is that we select highest energy node at each time of run.

Table-5 Node vs. Energy consumption (J)

\begin{tabular}{|l|l|l|}
\hline No. Of nodes & Base technique & Proposed work \\
\hline 20 & 191 & 154.7 \\
\hline 40 & 373 & 354.9 \\
\hline 60 & 545.9 & 546 \\
\hline 80 & 737 & 728 \\
\hline 100 & 919 & 910 \\
\hline
\end{tabular}

Table-6 Nodes vs. Packet delivery ratio

\begin{tabular}{|l|l|l|}
\hline No. Of nodes & Base technique & Proposed work \\
\hline 20 & 67.1523 & 50.2987 \\
\hline 40 & 81.9862 & 50.5025 \\
\hline 60 & 70.4435 & 53.5024 \\
\hline 80 & 61.8001 & 54.0142 \\
\hline 100 & 61.0501 & 57.7145 \\
\hline
\end{tabular}

Packet delivery ratio is calculated by the ratio of data packets which is received by the destinations to those generated by the sources. Mathematically, it can be calculated as:

\section{$\mathrm{PDR}=\mathrm{S} 1 / \mathrm{S} 2$}

Where, S1 defines as the summation of data packets that are received by each destination and S2 defines as the summation of data packets which is generated by each source. As we can see in above table the delay in packet is less in proposed algorithm as compared to previous algorithm given.

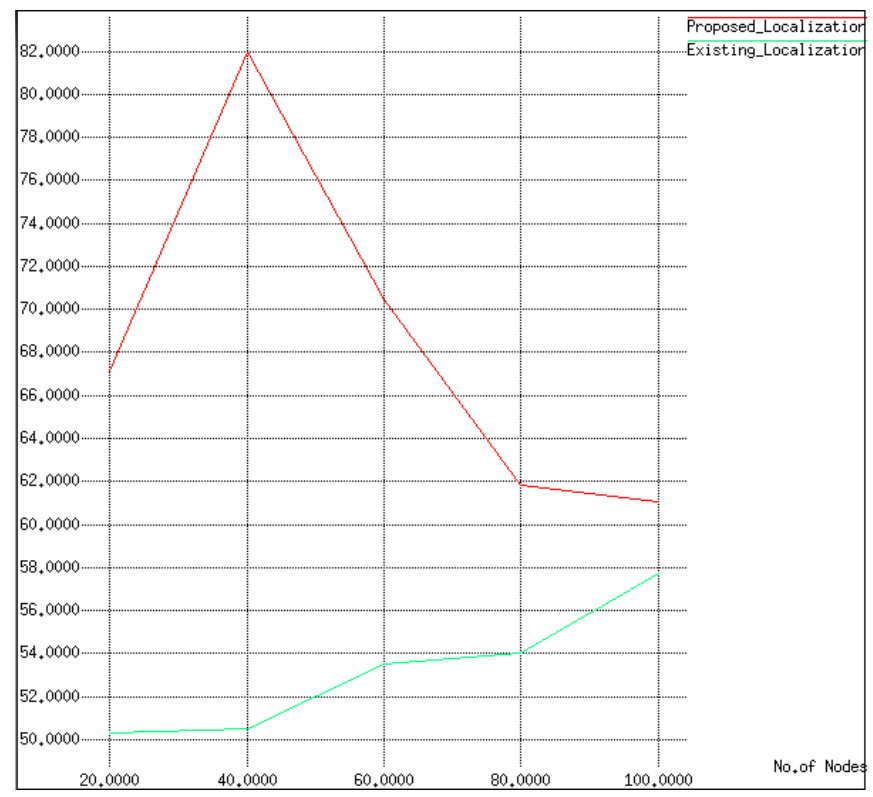

XGraph-3 Nodes vs. Packet delivery ratio

\section{CONCLUSION AND FUTURE ScOPE}

An event driven Localization scheme for distributed WSN is proposed with the assistance of the network simulator (NS-2) which evaluates value on the basis of an energy model and the localization ratio on different number of nodes that are deployed in underwater networks. The future scope of this research is to improve the threshold, energy consumption, Packet delivery ratio of nodes in 3D environment.

The main conclusions of the study may be presented in a short Conclusion Section. In this section, the author(s) should also briefly discuss the limitations of the research and Future Scope for improvement.

\section{REFERENCES}

[1] Juan Manuel Martinez," Planar localization of radio frequency or Acoustic sources with two parameters" presented at the 4th International Electronic Conference on Sensors and Applications, leganas, Spain.

[2] Guangjie Han, Jinfang Jiang, Ning Sun, and Lei Shu, 'Secure communication for underwater acoustic sensor networks"ISSN 0163-6804, volume 8, IEEE JOURNALS..

[3] Miguel Ardid illium, "Acoustic Transmitters for Underwater Neutrino Telescopes In Mathematical and Statistical Sciences", ISSN 1424-8220 mdpi journals.

[4] Mukesh Beniwal, "Localization Techniques and Their Challengesin “ Underwater Wireless Sensor networks", Vol. 5 (3) , 2014,4706-4710, I SSN 0975-9646 International Journal of Computer Science and Information Technologies.

[5] Emad Felemban," Underwater Sensor Network Applications:A Comprehensive Survey" Volume 2015, International Journal of Distributed Sensor Networks. 
[6] Pallavi G, " A recursive approach for distributed networks in UWSN" ISSN 2395-0072, International Research Journal of Engineering and Technology.

[7] V. Nancy Priyanka, P. Kaythry, R. Kishore."Performance analysis of Recursive Luby transform codes for error correction in underwater sensor network", 2017 International Conference on Wireless Communications, Signal Processing and Networking, (WiSPNET), 2017.

[8] Leena Pal, Pradeep Sharma, Netram Kaurav and Shivlal Mewada, "Performance Analysis of Reactive and Proactive Routing Protocols for Mobile Ad-hoc-Networks", International Journal of Scientific Research in Network Security and Communication, Vol.1, Issue.5, pp.1-4, 2013

[9] Pedram Vahdani Amoli. "An Overview on Current Researches on Underwater Sensor Networks: Applications, Challenges and Future Trends", International Journal of Electrical and Computer Engineering (IJECE), 2016.

[10] Preeti Sachan. "Authenticated Routing for Ad-Hoc On-Demand Distance Vector Routing Protocol", Communications in Computer And Information Science, 2011.

[11] Han, Guangjie, Jinfang Jiang, Ning Sun, and Lei Shu. "Secure communication for underwater acoustic sensor networks", IEEE Communications Magazine, 2015.

[12] Rajendra Aaseri ," Trust Value Algorithm: Approach Against Packet Drop Attack In Wireless Adhoc Networks", International Journal of Network Security \& Its Applications (IJNSA), Vol.5, No.3, May 2013.

[15] R. Nathiya, S.G. Santhi, "Energy Efficient Routing with Mobile Collector in Wireless Sensor Networks (WSNs)", International Journal of Computer Sciences and Engineering, Vol.2, Issue.2, pp.36-43, 2014. 